\title{
Protective Effect of Biological Osmolytes against Heat- and Chaotropic Agent-Induced Denaturation of Bacillus licheniformis $\gamma$-Glutamyl Transpeptidase
}

\author{
Huei-Fen $\mathrm{Lo}^{1 \dagger}$, Meng-Chun $\mathrm{Chi}^{2 \dagger}$, Min-Guan Lin $^{3}$, Yuan-Gin Lan ${ }^{2}$, Tzu-Fan Wang ${ }^{2 *}$, and Long-Liu Lin ${ }^{2 *}$ \\ ${ }^{1}$ Department of Food Science and Technology, Hungkuang University, 1018 Taiwan Boulevard, Shalu District, Taichung City 43302, Taiwan \\ ${ }^{2}$ Department of Applied Chemistry, National Chiayi University, 300 Syuefu Road, Chiayi City 60004, Taiwan \\ ${ }^{3}$ Institute of Molecular Biology, Academia Sinica, Nangang District, Taipei City 11529, Taiwan
}

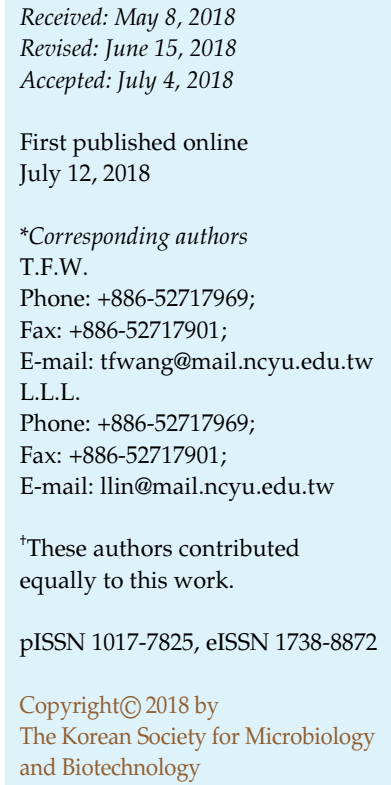

In the present study, the stabilizing effect of four different biological osmolytes on Bacillus licheniformis $\gamma$-glutamyl transpeptidase (BlGGT) was investigated. BlGGT appeared to be stable under temperatures below $40^{\circ} \mathrm{C}$, but the enzyme retained less than $10 \%$ of its activity at $60^{\circ} \mathrm{C}$. The tested osmolytes exhibited different degrees of effectiveness against temperature inactivation of BlGGT, and sucrose was found to be the most effective among these. The use of circular dichroism spectroscopy for studying the secondary structure of BlGGT revealed that the temperature-induced conformational change of the protein molecule could be prevented by the osmolytes. Consistently, the molecular structure of the enzyme was essentially conserved by the osmolytes at elevated temperatures as monitored by fluorescence spectroscopy. Sucrose was further observed to counteract guanidine hydrochloride ( $\mathrm{GdnHCl}$ and urea-induced denaturation of BlGGT. Taken together, we observed evidently that some well-known biological osmolytes, especially sucrose, make a dominant contribution to the structural stabilization of BlGTT.

Keywords: $\gamma$-Glutamyl transpeptidase, Bacillus licheniformis, osmolyte, sucrose, protein stabilization

\section{Introduction}

Most proteins are sensitive to changes in their surrounding environments, and any significant perturbation in thermodynamic conditions can definitely cause alterations in protein structure that consequently lead to a partial or complete loss of biological function [1]. Living organisms are known to adapt to such perturbations through an accumulation of high concentrations of organic molecules (biological osmolytes), which can increase the inherent stability of cellular proteins without compromising their native functionality [2]. A previous report has indicated that biological osmolytes are one of the most potent stabilizers for many disease-associated proteins and are capable of reversing their misfolding and/or aggregation events [3]. Beyond that, biological osmolytes can also modulate the molecular activity of heat-shock proteins probably due to the promotion of local refolding within the chaperone molecules [4].

Carbohydrates, methylamines, and amino acids are three major classes of biological osmolytes [5]. These biological substances can be further categorized into (i) osmolytes, such as trim ethylamine $N$-oxide (TMAO), sarcosine, sorbitol, sucrose and trehalose, that stabilize proteins by raising free energy of both native and denatured states, (ii) osmolytes, such as, glycine, betaine, proline and glycerol, that only moderately enhance the thermodynamic stability of proteins, (iii) denaturing osmolytes, such asurea, and (iv) counteracting osmolytes, such as the mixture of urea and TMAO) [5]. Over the last decades, an increasing number of studies have broadened the diverse functions of biological osmolytes pertaining to solubility of proteins [6-10], expression 
enhancement of enzymes [11, 12], and protection of living organisms against extreme environmental conditions [13-16].

$\gamma$-Glutamyl transpeptidase (GGT, E.C. 2.3.2.2) catalyzes the transfer of a $\gamma$-glutamyl group from $\gamma$-glutamyl donors to an acceptor that may be water, an amino acid, or a peptide [17]. The initial steps in the reaction catalyzed by GGT enzymes are the cleavage of the $\gamma$-carbonyl linkage of $\gamma$-glutamyl substrates and the formation of a transient enzyme-substrate (ES) complex [18, 19]. In the ES complex, the $\gamma$-carbon of $\gamma$-glutamyl substrates forms an acyl bond with the side-chain nucleophile of the catalytic Thr of GGT enzymes [20-22]. Hydrolysis of the ES complex occurs when a water molecule undergoes a nucleophilic attack at the carbonyl carbon of the esterified enzyme [20, 21]. However, in the presence of suitable L-amino acids or peptides, the transpeptidation reaction may actually proceed through intermolecular acylation to resolve the ES complex into new $\gamma$-glutamyl compounds [20]. Thus, a straightforward route towards the synthesis of a variety of $\gamma$-glutamyl compounds can be established by using GGT and different types of $\gamma$-glutamyl acceptors. In fact, GGT enzymes have been applied to the biocatalytic synthesis of a number of $\gamma$-glutamyl compounds with great industrial potential [23-26].

Biocatalytic synthesis of diverse types of chemical and biological compounds has become a promising way in chemical and pharmaceutical industries just because the biochemical process usually leads to a profound reduction in process time, number of reaction steps, and amount of waste [27]. Most importantly, biocatalytic processes always offer the most powerful approach to produce enantiomerically pure compounds through high regioselectivity, streoselectivity, and chemoselectivity of employed enzymes [28]. Although enzyme applications are being developed in full swing [29, 30], breakthroughs are still needed to address their weaknesses in maintaining activities during biocatalytic processes [31]. Osmolyte-mediated stabilization of enzymes may provide a potentially viable solution to overcome the instability problem [32]. Therefore, the objective of the current study is to explore the effect of four different biological osmolytes on the enzymatic activity and structural stability of Bacillus licheniformis GGT (BlGGT). The stabilizing effect of the tested osmolytes on BlGGT may be helpful in industrial applications of this enzyme.

\section{Materials and Methods}

\section{Gene Expression and Enzyme Purification}

For the expression of recombinant BlGGT, a single colony of
E. coli M15 cells harboring pQE-BlGGT [33] was inoculated into $10 \mathrm{ml}$ of Luria-Bertani (LB) broth containing ampicillin $(100 \mu \mathrm{g} / \mathrm{ml})$ and kanamycin $(25 \mu \mathrm{g} / \mathrm{ml})$, and the bacterial culture was incubated aerobically at $37^{\circ} \mathrm{C}$ for overnight. Then, $1 \mathrm{ml}$ of the overnight culture was transferred into $100 \mathrm{ml}$ of the antibioticcontaining LB broth and grown at $37^{\circ} \mathrm{C}$. Once an $\mathrm{A}_{600}$ of $0.6-0.8$ was reached, the bacterial culture was shifted to $28^{\circ} \mathrm{C}$ and induced for $6 \mathrm{~h}$ by the addition of isopropyl thio- $\beta$-D-galactoside to a final concentration of $100 \mu \mathrm{M}$. The induced cells were harvested by centrifugation and washed once with $50 \mathrm{mM}$ phosphate buffer (pH 7.0). The harvested cells were subsequently lysed by sonication and the resultant mixture was cleared of precipitates by centrifugation. Thereafter, the recombinant enzyme was purified from the supernatant by affinity chromatography using a nickelchelating column (Qiagen). The purity of BlGGT was monitored by resolution of the eluted sample on $12 \%$ SDS-polyacrylamide gel in Tris-glycine buffer followed by staining for $12 \mathrm{~h}$ with Comassie Brilliant Blue G-250. Protein concentration was determined by a quantitative Coomassie dye-binding assay (BioRad) using bovine serum albumin as a concentration standard.

\section{Activity Assay}

The transpeptidation activity of BlGGT was determined by a colorimeter assay as described previously [33]. Briefly, BlGGT and the reaction components $(1.25 \mathrm{mM} \mathrm{L}-\gamma$-glutamyl-p-nitroanilide, $30 \mathrm{mM}$ Gly-Gly, $75 \mathrm{mM} \mathrm{NaCl}$, and $20 \mathrm{mM}$ Tris- $\mathrm{HCl}$ buffer; $\mathrm{pH}$ 9.0) were warmed up separately at $40^{\circ} \mathrm{C}$ for $10 \mathrm{~min}$. The catalytic action was initiated by adding suitably diluted enzyme solution to the reaction components and enough distilled water to bring the final volume up to $1 \mathrm{ml}$. The mixture was then incubated at $40^{\circ} \mathrm{C}$ for $10 \mathrm{~min}$ and the enzymatic reaction was stopped chemically by adding $100 \mu \mathrm{l}$ of acetic acid $(3.5 \mathrm{~N})$. Release of $p$-nitroaniline from L- $\gamma$-glutamyl- $p$-nitroanilide was determined by monitoring the absorbance change at $410 \mathrm{~nm}$ using a UV-vis spectrophotometer. One unit of BlGGT activity is defined as the amount of enzymes that catalyze the conversion of $1 \mu \mathrm{mol}$ of substrate per min under the assay conditions.

\section{Effect of Osmolytes on the Thermal Stability of BlGGT}

To investigate the effect of glycerol, sorbitol, sucrose and TMAO on the thermal stability of BlGGT, the deactivation was carried out by incubating the enzyme-osmolyte samples (a final enzyme concentration of $50 \mu \mathrm{g} / \mathrm{ml}$ and a final osmolyte concentration of $2 \mathrm{M}$ ) at various temperatures of $30^{\circ} \mathrm{C}, 40^{\circ} \mathrm{C}, 45^{\circ} \mathrm{C}$, $50^{\circ} \mathrm{C}, 55^{\circ} \mathrm{C}, 60^{\circ} \mathrm{C}, 65^{\circ} \mathrm{C}, 70^{\circ} \mathrm{C}, 80^{\circ} \mathrm{C}$, and $90^{\circ} \mathrm{C}$ for $20 \mathrm{~min}$. The heated samples were taken out after different intervals of time and cooled immediately in ice water. The residual activity was measured using standard assay conditions as described above. An experimental control was simultaneously performed using osmolyte-free enzyme solution.

\section{Circular Dichroism (CD) and Fluorescence Analyses}

Protein secondary structure analyses were carried out at two 
different temperatures $\left(40^{\circ} \mathrm{C}\right.$ and $\left.60^{\circ} \mathrm{C}\right)$ using a Jasco J-815 spectropolarimeter (Jasco, Japan) equipped with a cell holder thermostatically controlled by circulating water from a bath. The instrument was operated with Jasco's Spectra Manager software and all the measurements were conducted under a nitrogen flow of $10 \mathrm{l} / \mathrm{min}$. The CD spectra were recorded between 190 and $250 \mathrm{~nm}$ with a 0.2-nm step resolution and a scanning speed of $20 \mathrm{~nm} / \mathrm{min}$. All enzyme samples were diluted with $20 \mathrm{mM}$ Tris$\mathrm{HCl}$ buffer ( $\mathrm{pH}$ 9.0) to give a protein concentration of approximately $7.6 \mu \mathrm{M}$. After CD measurements, background contribution of $20 \mathrm{mM}$ Tris- $\mathrm{HCl}$ buffer ( $\mathrm{pH}$ 9.0) was subtracted from each spectrum to normalize it. The mean molar residue ellipticity $(\theta)$ was calculated according to the following formula: $[\theta]=\theta_{\mathrm{obs}}$ (in mdeg)/(molar concentration of the enzyme $\times$ optical path length $x$ number of amino acid residues in the enzyme) [34]. The CD intensity is defined in terms of molar residue ellipticity (MRE) with a unit of $\mathrm{deg} \cdot \mathrm{cm}^{2} \cdot \mathrm{dmol}^{-1}$.

Heat-induced unfolding of BlGGT was performed by monitoring the change in ellipticity at a fixed wavelength of $222 \mathrm{~nm}$. Prior to CD measurements, the purified BlGGT was mixed with an appropriate amount of $20 \mathrm{mM}$ Tris- $\mathrm{HCl}$ buffer ( $\mathrm{pH}$ 9.0) and various amounts of each osmolyte stock to generate enzyme solutions with a protein concentration of $\sim 12.4 \mu \mathrm{M}$. Enzyme samples were heated at a constant scanning rate of $1^{\circ} \mathrm{C} / \mathrm{min}$. Ellipticity values at $222 \mathrm{~nm}$ wavelength were specifically extracted from the $\mathrm{CD}$ spectra to determine the melting temperature $\left(T_{\mathrm{m}}\right)$ of enzyme samples. The transition temperatures were calculated by fitting the CD data with Eq. (1) [35].

$$
\theta_{222}=\frac{\theta_{N}+\theta_{U} \cdot \exp \left[-\frac{\Delta H_{U}}{R T} \cdot\left(1-\frac{T}{T_{m}}\right)+\frac{\Delta C_{P U}}{R T} \cdot\left(T \ln \left(\frac{T}{T_{m}}\right)+T_{m}-T\right)\right]}{1+\exp \left[-\frac{\Delta H_{U}}{R T} \cdot\left(1-\frac{T}{T_{m}}\right)+\frac{\Delta C_{P U}}{R T} \cdot\left(T \ln \left(\frac{T}{T_{m}}\right)+T_{m}-T\right)\right]}
$$

where $\theta_{222}$ is the relative ellipticity at $222 \mathrm{~nm}$ wavelength; $\theta_{\mathrm{N}}$ and $\theta_{U}$ represent the fraction of enzyme present in the native and unfolded states; $\Delta H_{U}$ is the free enthalpy for BlGGT unfolding; $\Delta C_{P U}$ is the heat capacity for BlGGT unfolding; and $R$ represents the universal gas constant.

Counteraction of sucrose against urea-induced denaturation of BlGGT was performed by adding the sugar osmolyte into enzyme samples of $12.4 \mu \mathrm{M}$ in $20 \mathrm{mM}$ Tris- $\mathrm{HCl}$ buffer ( $\mathrm{pH} 9.0$ ) containing the chaotropic agent to molar ratios of $0.5: 1,1: 2,1.5: 3$, and 2:4. The denaturation process of $B l$ GGT was determined by recording the CD signal at $222 \mathrm{~nm}$. The change in the $T_{\mathrm{m}}$ value was used to monitor the unfolding of BlGGT.

Steady-state emission spectra were recorded at ambient temperature on a JASCO FP-6500 fluorescence spectrophotometer (Japan) with an excitation wavelength of $295 \mathrm{~nm}$ using a 0.1-ml quartz cuvette. The emission spectra of BlGGT and enzymeosmolyte samples with a concentration of approximately $16.2 \mu \mathrm{M}$ were measured from 305 to $500 \mathrm{~nm}$ at a scanning speed of $240 \mathrm{~nm} / \mathrm{min}$. Thereafter, the emission spectra ofBlGGT and enzyme-osmolyte samples were individually corrected for the contribution of $20 \mathrm{mM}$ Tris-HCl buffer ( $\mathrm{pH}$ 9.0). GdnHCl-induced denaturation of $B l G G T$ was determined by initially recording fluorescence spectra of protein samples in $20 \mathrm{mM}$ Tris- $\mathrm{HCl}$ buffer (pH 9.0) containing $3 \mathrm{M} \mathrm{GdnHCl}$. Counteraction of sucrose against GdnHCl-induced denaturation of BlGGT was performed by individually adding the sugar osmolyte into the aforementioned samples to a final concentration of $2 \mathrm{M}$. Eventually, the maximal peak of the fluorescence spectrum and the change in the fluorescence intensity were used to monitor the unfolding process of each sample. Both the emission wavelength shift and the change in fluorescence intensity were analyzed together to calculate the average emission wavelength (AEW) $(\lambda)$ of each sample according to Eq. (2) [36].

$$
<\lambda>=\frac{\sum_{i=\lambda_{1}}^{\lambda_{N}}\left(F_{i} \cdot \lambda_{i}\right)}{\sum_{i=\lambda_{1}}^{\lambda_{N}} F_{i}}
$$

in which $F_{\mathrm{i}}$ represents the fluorescence intensity at the specific emission wavelength $\left(\lambda_{\mathrm{i}}\right)$.

\section{Results}

\section{Prevention of $B l G G T$ from Heat-Induced Inactivation by Biological Osmolytes}

The transpeptidation activity of BlGGT was examined in response to a number of biological osmolytes and different temperature conditions. As shown in Fig. 1A, the enzyme was inactivated in a temperature-dependent manner regardless of presence or absence of osmolytes. It is noteworthy that all tested osmolytes were able to protect $B l$ GGT against thermal inactivation. Incubation of BlGGT at $60^{\circ} \mathrm{C}$ lost more than $85 \%$ of the enzymatic activity, but this situation was greatly improved by the addition of biological osmolytes. Among the employed osmolytes, the protective effect of sucrose on the thermal inactivation of BlGGT was more obvious than others (Fig. 1A).

The molecular properties and structure-function relationships of BlGGT have been extensively studied in our laboratory [37-40]. The results of these investigations clearly demonstrate that the recombinant enzyme has an optimal activity at $40^{\circ} \mathrm{C}$. It is noteworthy that BlGGT was quite stable at $40^{\circ} \mathrm{C}$ even in the absence of $2 \mathrm{M}$ sucrose (Fig. 1B). However, a dramatic loss in GGT activity was observed after incubating the enzyme at either $60^{\circ} \mathrm{C}$ or $70^{\circ} \mathrm{C}$ for $10 \mathrm{~min}$. This phenomenon was greatly improved by $2 \mathrm{M}$ sucrose, with more than $80 \%$ retention of the enzymatic activity over an incubation time of $6 \mathrm{~h}$ (Fig. 1B). The stabilizing effect will be very valuable for the BlGGT- 

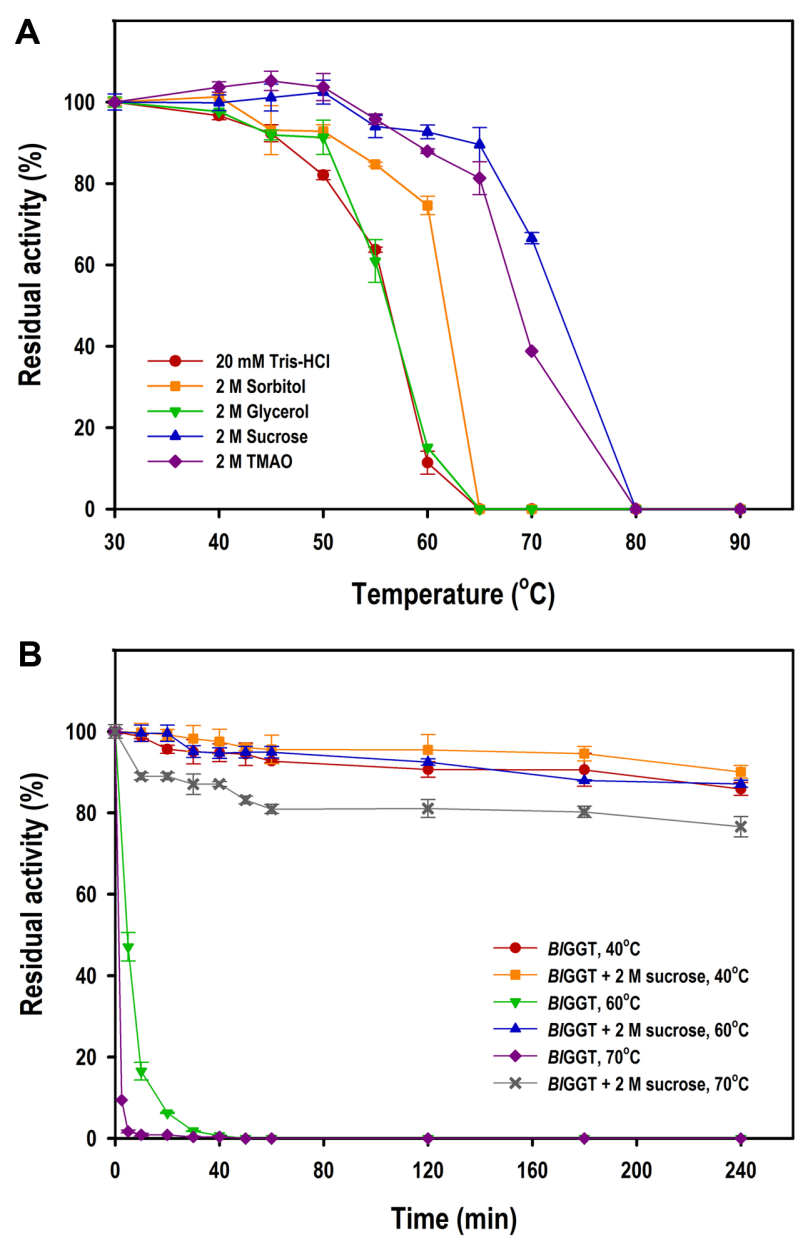

Fig. 1. Stabilization of BlGGT by biological osmolytes.

(A) Effect of four different osmolytes on the thermal stability of the enzyme. (B) Thermal inactivation curves of BlGGT in the absence and presence of $2 \mathrm{M}$ sucrose. Aliquots of each sample were immediately cooled and assayed for GGT activity under the standard assay conditions.

mediated synthesis of $\gamma$-glutamyl compounds at elevated temperatures. Advantages for a biocatalytic process operated at high temperatures have been documented [31, 41]. Besides, the stabilizing effect definitely renders the BlGGT-catalyzed process more economical due to the high cost of enzyme production and purification [31].

Our previous studies have shown that the CD spectrum of BlGGT exhibits two strong peaks of negative ellipticity at 208 and $222 \mathrm{~nm}$ [37, 42], an indication of substantial $\alpha$-helical content. These spectrometric characteristics were essentially conserved in enzyme-osmolyte samples when the denaturation temperature was set at $40^{\circ} \mathrm{C}$ (Fig. 2A). However, upon heat treatment of $\mathrm{BlGGT}$ at $75^{\circ} \mathrm{C}$ for $20 \mathrm{~min}$,
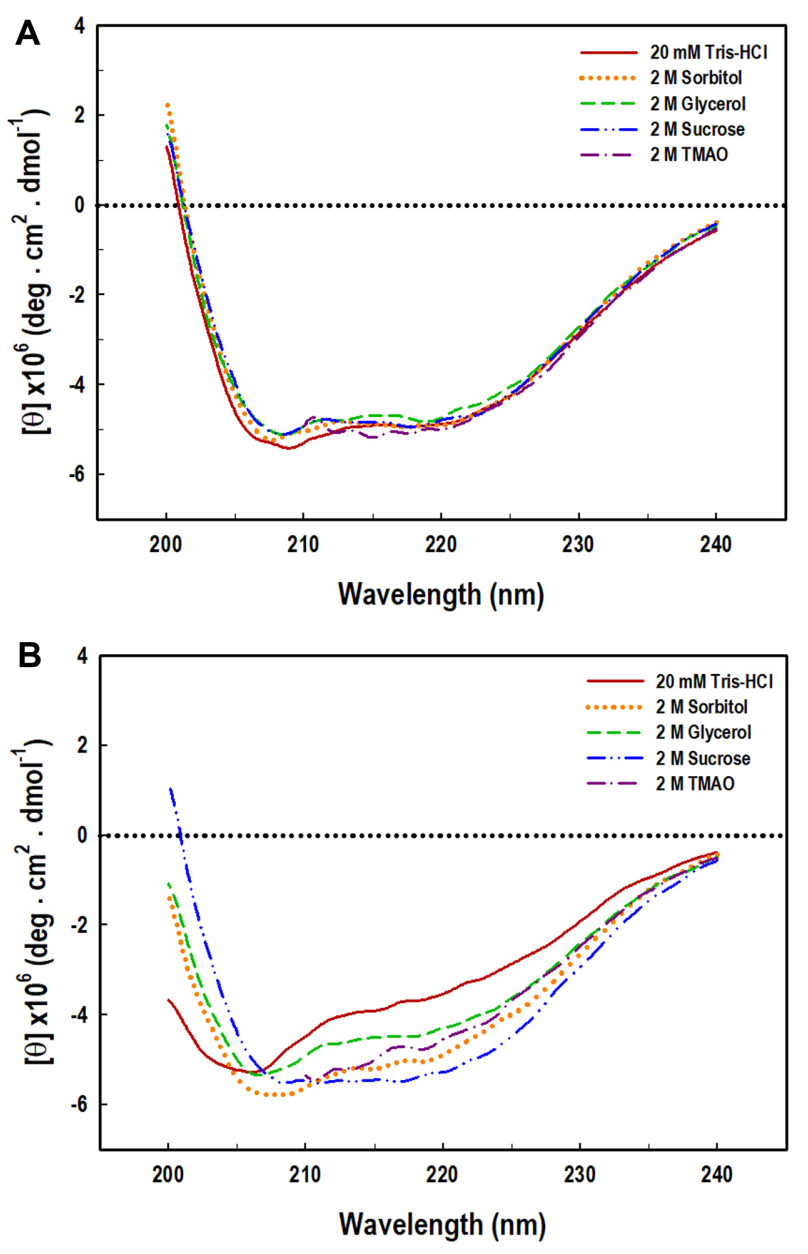

Fig. 2. Far-UV CD spectra of BlGGT in the absence and presence of four different biological osmolytes.

The spectra were recorded over a wavelength range of 190-250 $\mathrm{nm}$ at temperatures of $40^{\circ} \mathrm{C}(\mathbf{A})$ and $75^{\circ} \mathrm{C}(\mathbf{B})$.

the CD signal at $222 \mathrm{~nm}$ was profoundly diminished in osmolyte-free samples (Fig. 2B). It is interesting to note that the representative peaks for the $\alpha$-helix of BlGGT were mostly reserved in the presence of biological osmolytes. Thus, the tested osmolytes, especially sucrose, can apparently protect BlGGT against heat-induced conformational change.

The conformational stability of BlGGT was also investigated by recording the molar ellipticity at $222 \mathrm{~nm}$. Visual inspection of the transition curves indicated that the thermal unfolding of all tested samples followed a twostate process (Fig. 3). BlGGT exhibited one well-defined unfolding transition of $61.1^{\circ} \mathrm{C}$. Notably, in the presence of $2 \mathrm{M}$ osmolytes, the $T_{\mathrm{m}}$ value was individually increased to $64.9^{\circ} \mathrm{C}$ (sorbitol), $64.8^{\circ} \mathrm{C}$ (glycerol), $79.7^{\circ} \mathrm{C}$ (sucrose), and 


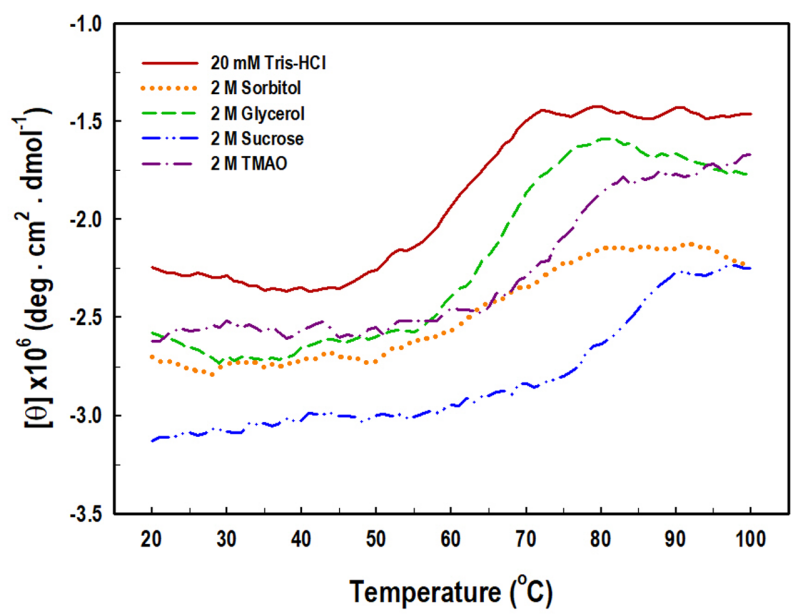

Fig. 3. Effect of biological osmolytes on the thermal stability of BlGGT.

The protein samples in $20 \mathrm{mM}$ Tris- $\mathrm{HCl}$ buffer ( $\mathrm{pH}$ 9.0) were monitored with the CD signal at $222 \mathrm{~nm}$.

$73.3^{\circ} \mathrm{C}$ (TMAO). This observation allows us to confirm that the employed osmolytes are able to protect BlGGT against temperature-induced denaturation.

\section{Counteraction of GdnHCl-Induced Denaturation of BlGGT by Sucrose}

The chaotropic agent $\mathrm{GdnHCl}$ is commonly used as a protein denaturant to generally bring about unfolding of proteins by disrupting their secondary and tertiary structures [43]. In this respect, unfolding of BlGGT at different concentrations of $\mathrm{GdnHCl}$ has already been performed [44]. The enzyme starts to unfold at a denaturant concentration of $1.92 \mathrm{M}$ and reaches an unfolded intermediate, $[\mathrm{GdnHCl}]_{0.5, \mathrm{~N}-\mathrm{U}}$, at $3.07 \mathrm{M}$. Based on this information, the transpeptidation activity of BlGGT was determined in the presence of $3 \mathrm{MGdnHCl}$ to examine the correlation between conformational dynamics and enzyme catalysis. As shown in Fig. 4, $\mathrm{GdnHCl}$ displayed a timedependent inhibitory effect on the catalytic activity of BlGGT. Upon the incubation of BlGGT in aqueous solutions of $3 \mathrm{M} \mathrm{GdnHCl}$ for $25 \mathrm{~min}$, the enzyme retained $15.2 \% \pm 1.5$ of its initial activity. The transpeptidation activity was almost abolished when the incubation time was extended to more than $6 \mathrm{~h}$. It is quite interesting to note that the functional inactivation of the enzyme by $3 \mathrm{M} \mathrm{GdnHCl}$ was tremendously inhibited in the presence of 1.5 and $2.0 \mathrm{M}$ sucrose (Fig. 4). This observation clearly indicates the potent ability of this common sugar to counteract the $\mathrm{GdnHCl}$-induced denaturation.

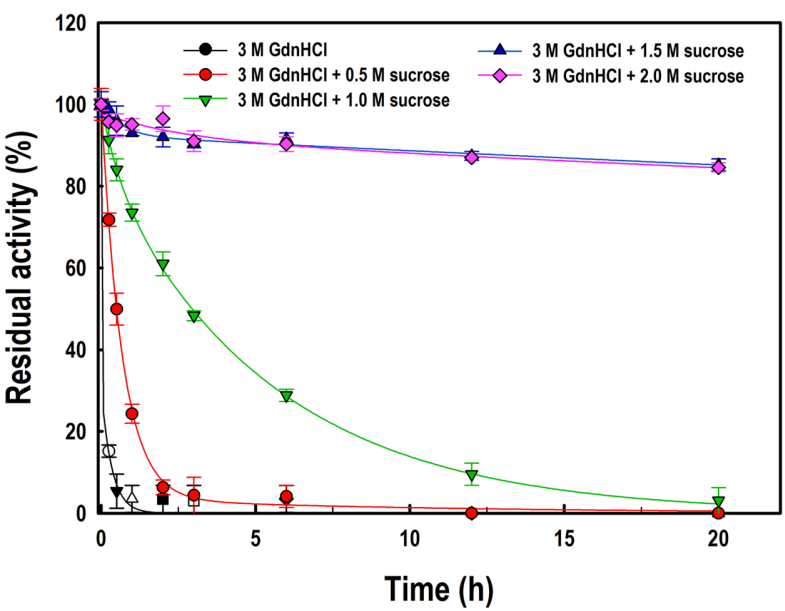

Fig. 4. Protective effect of sucrose against $\mathrm{GdnHCl}$-induced inactivation of BlGGT.

The purified enzyme was mixed with $3.0 \mathrm{M} \mathrm{GdnHCl}$ and different concentrations of sucrose. Aliquots of each sample were withdrawn at desired time intervals to test the residual activity.

The protective effect of sucrose against GdnHCl-induced denaturation of $B l G G T$ was further verified by $C D$ and fluorescence spectroscopy. As shown in Fig. 5A, the negative ellipticity of GdnHCl-treated BlGGT was profoundly decreased within the first $30 \mathrm{~min}$ in the absence of sucrose, reflecting a greatly diminished native secondary structure of the enzyme. A similar phenomenon was observed with $0.5 \mathrm{M}$ sucrose/ $3 \mathrm{M}$ GdnHCl-treated BlGGT. However, the decrease in the negative ellipticity of GdnHCl-treated BlGGT was slowed down in the presence of $1.0 \mathrm{M}$ sucrose. GdnHCl-induced secondary structural changes of BlGGT were almost inhibited by 1.5 and 2. $0 \mathrm{M}$ sucrose even after prolonged incubation (Fig. 5A). Therefore, the protective effect of sucrose on the secondary structure of BlGGT is very obvious at sugar concentrations of above $1.5 \mathrm{M}$.

A valuable feature of intrinsic protein fluorescence is the high sensitivity of tryptophan to its local environment. Changes in the fluorescence spectra of proteins often occur in response to substrate binding, subunit association, conformational transitions, or denaturation [45]. Therefore, AEW that reports on the changes in both emission wavelength and fluorescence intensity can be suitably used to monitor the conformational change of BlGGT. As shown in Fig. 5B, upon the treatment of $\mathrm{BlGGT}$ with $3 \mathrm{M} \mathrm{GdnHCl}$, the AEW value seemed to level off over a relatively short period of time. In the presence of $0.5 \mathrm{M}$ sucrose, the rate of conformational change for GdnHCl-treated BlGGT increased linearly for the first $20 \mathrm{~min}$ and then began to level off after 

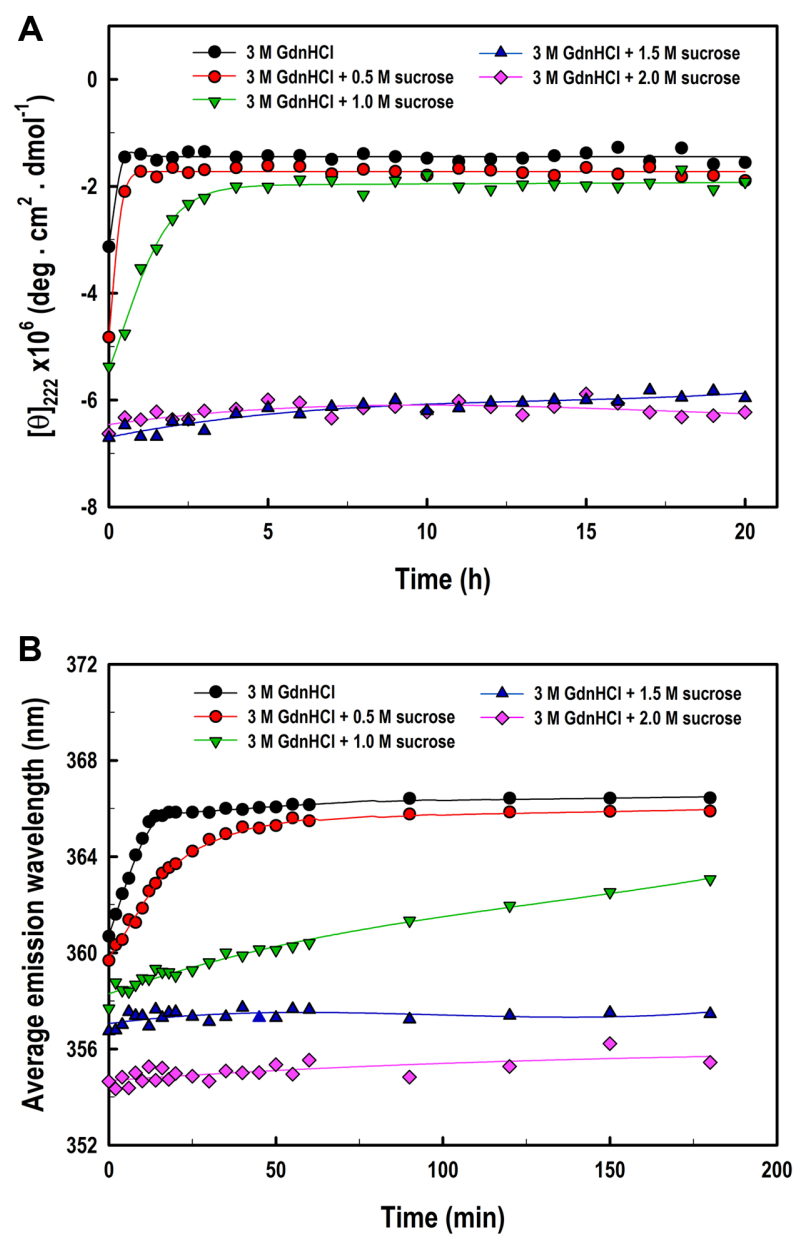

Fig. 5. Counteraction of $\mathrm{GdnHCl}$-induced denaturation of BlGGT by sucrose as monitored changes in the negative ellipticity at $222 \mathrm{~nm}(\mathbf{A})$ and in the AEW value (B).

50 min. Consistent with the above-mentioned CD data, there was no significant change in the AEW value of GdnHCl-treated BlGGT in the presence of 1.5 and $2 \mathrm{M}$ sucrose (Fig. 5B). Apparently, the results of $\mathrm{CD}$ and fluorescence measurements confirm the counteracting ability of sucrose against the $\mathrm{GdnHCl}$-induced denaturation of BlGGT.

\section{Sucrose Offsets Urea's Effect on BlGGT Structure}

Fig. 6 shows the change in $T_{\mathrm{m}}$ values, which correspond to the transition of BlGGT to the unfolded state, as a function of sucrose or urea. It can be seen that sucrose increased the $T_{\mathrm{m}}$ value of $B l \mathrm{GGT}$ with different increments of osmolyte concentrations (changing from $61.1^{\circ} \mathrm{C}$ in the absence of osmolyte to $79.7^{\circ} \mathrm{C}$ in the presence of $2.0 \mathrm{M}$ sucrose). However, urea lowered the $T_{\mathrm{m}}$ value of $B l \mathrm{GGT}$ by

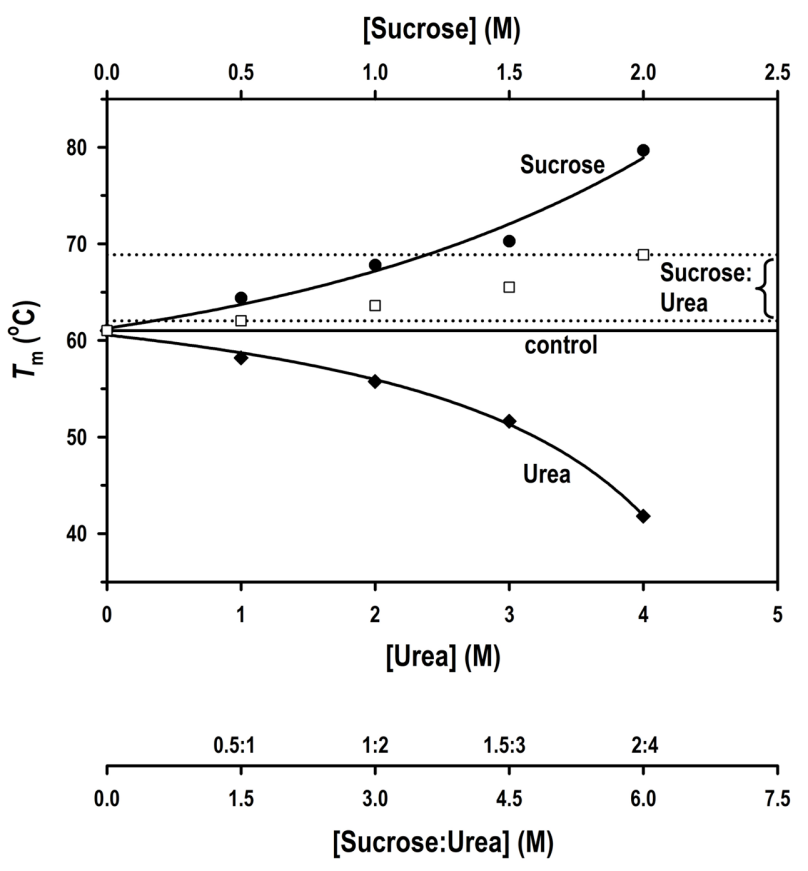

Fig. 6. Counteraction of urea-induced denaturation of BlGGT by sucrose.

The point $61.1^{\circ} \mathrm{C}$ is the control, which represents the BlGGT transition temperature in buffer solution (the solid line). The dashed lines show the region of sucrose + urea.

a concentration-dependent manner (changing from $61.1^{\circ} \mathrm{C}$ in the absence of urea to $41.8^{\circ} \mathrm{C}$ in the presence of urea). The experimental results explicitly indicate that the sugar osmolyte increases the transition temperatures and keeps the folding form beyond $79^{\circ} \mathrm{C}$, which is obviously higher than the control. Fig. 6 also depicts that the $T_{\mathrm{m}}$ values of BlGGT in sucrose-plus-urea mixtures (sucrose and urea at molar ratios of $0.5: 1,1: 2,1.5: 3$, and 2:4) stably increased and these mixtures had almost the same trend as sucrose-alone action on BlGGT. Based on the fact that sucrose-plus-urea mixtures substantially increase the $T_{\mathrm{m}}$ value of BlGGT, sucrose can indeed offset the urea-induced denaturation of the enzyme.

\section{Discussion}

Protein structure is stabilized mainly by a combination of hydrophobic interactions, hydrogen bonding and electrostatic interactions with additional contributions in particular proteins arising from metal complexing, cross-linking, and specific bindings of ions and cofactors. Some previous studies have shown that protein stability is essentially established through a balance between intramolecular 
interactions of protein functional groups and their interaction with solvent environment [46-48]. Accordingly, the effects of sugars and polyols on the thermal stability of proteins depend upon how they influence the overall balance of interactions. Our results clearly indicate that sucrose is the most effective osmolyte for preventing BlGGT from thermally induced functional inactivation (Fig. 1). As a naturally occurring sugar, sucrose has also been shown to increase the conformational stability of the native state of several globular proteins against temperature-induced denaturation [49-51]. Although the molecular-level mechanism of sucrose-mediated stabilization is not yet fully understood, the solvent-excluded volume effect may play a fundamental role to stabilize the $N$-state of globular proteins [52]

Biological osmolytes have been reported to push the reversible folding equilibrium of a protein toward natively folded conformation by raising the free energy of unfolded state [53]. As they predominantly affect the protein backbone, the balance between osmolyte-backbone interactions and amino acid side chain-solvent interactions is surely an important criterion of protein folding [53]. It is well known that the stabilizing osmolytes are preferentially excluded from the immediate vicinity of the protein surface, leading to the formation of a solvophobic interaction between peptide backbone and side chain on the protein surface and the protecting osmolytes [54]. The tendency to exclude osmolytes from the protein surface forces polypeptides to adopt a folded conformation with a minimum possible exposed surface area [55]. Thus, protein-osmolyte compatibility depends on the degree of freedom a protein backbone has to bury itself during the folding of polypeptides into a native-like conformation [56]. Because of their preferential hydration, biological osmolytes have been shown to increase the respective $T_{\mathrm{m}}$ values of many proteins [57-59]. Apparently, the tendency of increased $T_{m}$ value of BlGGT by biological osmolytes is consistent with previous studies. This allows us to conclude that the tested biological osmolytes, especially sucrose and TMAO, can efficiently protect BlGGT against temperature-induced denaturation.

Protein unfolding by the classical denaturants, such as $\mathrm{GdnHCl}$ and urea, has long been considered to arise from the favorable interactions of the chaotropic agents with the normally buried segments of proteins [60]. The basis of bimolecular interactions for the destabilization of chemical denaturants are generally attributed to either a direct ligand binding with the protein surface or the effect of denaturants on the structure and dynamic of water molecules [61, 62]. In our case, sucrose was found to counteract the GdnHCl-induced unfolding of BlGGT (Fig. 5). Some previous studies have already provided possible interpretations for the protective effect of sucrose on $\mathrm{GdnHCl}$-induced denaturation of proteins [63-66]. It has been shown that $\mathrm{GdnHCl}$ disrupts the structure of ordered water of a phosphoglycerate kinase and this conformational change can be counteracted by sucrose [63]. A comprehensive investigation of the sucrose-mediated effect on the $\mathrm{GdnHCl-induced} \mathrm{denaturation} \mathrm{of} \mathrm{proteins} \mathrm{further} \mathrm{reveals}$ that this sugar is able to inhibit conformation fluctuations within the native state of proteins as well as increase the free energy of unfolding [66]. Thus, the aforementioned elucidations might be appropriate to interpret the protective effect of sucrose on the GdnHCl-induced denaturation of BlGGT.

The evolutionary advantage of biological osmolyte systems is compatible with macromolecular structure at high and variable osmolyte concentrations without modifying the functionality of cellular proteins $[67,68]$. Although compatible osmolytes are largely accumulated to stabilize protein and enzyme systems, nature has not ignored the use of proteindestabilizing osmolytes to act as efficient osmoprotectants. Urea, a metabolic waste of living organisms, and amino acid osmolytes (for example, arginine, histidine, and lysine) are familiar compounds for use as an osmoprotectant [67, 68]. It has been reported that urea can disrupt the noncovalent interactions responsible for the molecular structure of proteins [69]. The denatured protein with exposed hydrophobic residues is probably stabilized by enclosure of its hydrophobic sectors in a cage-like structure, which minimizes the contact with the aqueous phase and the entropy loss of the system. Hua and her coworkers has proposed a direct interaction mechanism in which urea has a stronger dispersion interaction with proteins than water [70]. In order to counteract the denaturing effect of urea on proteins in vivo, living organisms or cells produce several types of biological osmolytes, such as TMAO [71], betaine [72], and polyols [67] to stabilize protein structure and to maintain its functionality under high levels of urea. Furthermore, biological osmolytes TMAO, betaine, and sarcosine have also been shown to counteract urea-induced denaturation of protein [73]. Giving the fact that urea-induced denaturation of BlGGT can be counteracted by sucrose, the experimental data thus provide more insights into osmolyte-mediated protein stabilization, and the findings are expected to further facilitate the practical use of this biological osmolyte on the recovery of recombinant 
proteins from inclusion bodies.

In conclusion, the potential of protective biological osmolytes as protein stabilizers has been further extended by this study. Our experimental results have shed some light on the structural alterations and loss of functionality of BlGGT upon exposure of this enzyme to heat and denaturants. Furthermore, sucrose-mediated stabilization of the BlGGT structure is surely helpful in the retention of enzymatic activity at elevated temperatures and in the presence of denaturants. From an economic viewpoint, the stabilization strategy is simpler and less expensive than other methods like protein engineering, chemical modification, and immobilization. A further advantage is that the sugar stabilizer could be added to the enzyme solution just when it is desired.

\section{Acknowledgment}

The authors are eternally grateful for the research grant (MOST106-2330-B-B-415-001) from the Ministry of Science and Technology of Taiwan. The funder has no role in either experimental design, data analysis and interpretation, decision to publish, nor preparation of the manuscript.

\section{Conflict of Interest}

The authors have no financial conflicts of interest to declare.

\section{References}

1. Závodszky P, Kardos J, Svingor A, Petsko GA. 1998. Adjustment of conformational flexibility is a key event in the thermal adaption of proteins. Proc. Natl. Acad. Sci. USA 95: 7406-7411.

2. Yancey PH, Clark ME, Hand SC, Bowlus RD, Somero GN. 1982. Living with water stress: evolution of osmolyte systems. Science 217: 1214-1222.

3. Lamitina T, Huang CG, Strange K. 2006. Genome-wide RNAi screening identifies protein damage as a regulator of osmoprotective gene expression. Proc. Natl. Acad. Sci. USA 103: 12173-12178.

4. Diamant S, Eliahu N, Rosenthal D, Goloubinoff P. 2001. Chemical chaperones regulate molecular chaperones in vitro and in cells under combined salt and heat stresses. J. Biol. Chem. 276: 39586-39591.

5. Judy E, Kishore N. 2016. Biological wonders of osmolytes: the need to know more. Biochem. Anal. Biochem. 5: 4.

6. Auton M, Rösgen J, Sinev M, Holthauzen LMF, Bolen DW. 2011. Osmolyte effects on protein stability and solubility: a balancing act between backbone and side-chains. Biophys. Chem. 159: 90-99.

7. Garcia-Manyes S, Dougan L, Fernández JM. 2009. Direct observation of an ensemble of stable collapsed states in the mechanical folding of ubiquitin. Proc. Natl. Acad. Sci. USA 106: 10540-10545.

8. Harries D, J Rösgen J. 2008. A practical guide on how osmolytes modulate macromolecular properties. Methods Cell. Biol. 84: 679-735.

9. Heyda J, Kožíšek M, Bednárova L, Thompson G, Konvalinka J, Vondrášek J, et al. 2011. Urea- and guanidinium induceddenaturation of a Trp-cage miniprotein. J. Phys. Chem. B 115: 8910-8924.

10. Politi R, Harries D. 2010. Enthalpically-driven peptide stabilization by protective osmolytes. Chem. Commun. 46: 6449-6451.

11. Rashid N, Thapliyal C, Chaudhuri P.2017. Osmolyteinduced enhancement of expression and solubility of human dihydrofolate reductase: an in vivo study. Int. J. Biol. Macromol. 103: 1044-1053.

12. Thapliyal C, Chaudhuri P. 2015. Effect of various osmolytes on the expression and functionality of zebrafish dihydrofolate reductase: an in vivo study. J. Proteins Proteomics 6: 211-218.

13. Costanzo JP. 2005. Cryoprotection by urea in a terrestrially hibernating frog. J. Exp. Biol. 208: 4079-4089.

14. Sakamoto A, Murata N. 2002. The role of glycine betaine in the protection of plants from stress: clues from transgenic plants. Plant Cell Environ. 25: 163-171.

15. Shimizu S, Smith PE. 2017. How osmolytes counteract pressure denaturation on a molecular scale. Chemphyschem 18: 2243-2249.

16. Yancey PH, Speers-Roesch B, Atchinson S, Reist JD, Majewski AR, Treberg JR. 2018. Osmolyte adjustments as a pressure adaptation in deep-sea chondrichthyan fishes: an intraspecific test in arctic skates (Amblyraja hyperborean) along a depth gradient. Physiol. Biochem. Zool. 91: 788-796.

17. Keillor JW, Castonguay R, Lherbet C. 2005. $\gamma$-Glutamyl transpeptidase substrate specificity and catalytic mechanism. Methods Enzymol. 401: 449-467.

18. Tate SS, Meister A. 1974. Interaction of $\gamma$-glutamyl transpeptidase with amino acids, dipeptides, and derivatives and analogs of glutathione. J. Biol. Chem. 249: 7593-7602.

19. Castonguay R, Lherbet C, Keillor JW. 2003. Kinetic studies of rat kidney $\gamma$-glutamyl transpeptidase deacylation reveal a general base-catalyzed mechanism. Biochemistry 42: 11504-11513.

20. Thompson GA, Meister A. 1976. Hydrolysis and transfer reactions catalyzed by $\gamma$-glutamyl transpeptidase: evidence for separate substrate sites and the high affinity of L-cysteine. Biochem. Biophys. Res. Commun. 71: 32-36.

21. Lherbet C, Keiller JW. 2004. Probing the stereochemistry of the active site of $\gamma$-glutamyl transpeptidase using sulfur derivatives of L-glutamic acid. Org. Biomol. Chem. 2: 238-245.

22. Han L, Hiratake J, Kamiyama A, Sakata K. 2007. Design, 
synthesis, and evaluation of $\gamma$-phosphonodiester analogues of glutamate as highly potent inhibitors and active site probes of $\gamma$-glutamyl transpeptidase. Biochemistry 46: 1432-1447.

23. Suzuki H, Yamada C, Kato K. 2007. $\gamma$-Glutamyl compounds and their enzymatic production using bacterial $\gamma$-glutamyl transpetidase. Amino Acids 32: 333-340.

24. Castellano I, Merlino A. 2012. $\gamma$-Glutamyl transpeptidases: sequence, structure, biochemical properties, and biotechnological applications. Cell. Mol. Life Sci. 69: 3381-3394.

25. Speranza G, Morelli CF. 2012. $\gamma$-Glutamyl transpeptidasecatalyzed synthesis of naturally occurring flavor enhancers. J. Mol. Catal. B Enzym. 84: 65-71.

26. $\mathrm{Mu} \mathrm{W}$, Zhang T, Jiang B. 2015. An overview of biological production of L-theanine. Biotechnol. Adv. 33: 335-342.

27. Wohlgemuth R. 2010. Biocatalysis-key to sustainable industrial chemistry. Curr. Opin. Biotechnol. 21: 713-724.

28. Nestl BM, Nebel BA, Hauer B. 2011. Recent progress in industrial biocatalysis. Curr. Opin. Chem. Biol. 15: 187-193.

29. Sarrouh B, Santos TM, Miyoshi A, Dias R, Azevedo V. 2012. Up-to-date insight on industrial enzymes applications and global market. J. Bioprocess Biotechniq. S4: 002.

30. Singh R, Kumar M, Mittal A, Mehta PK. 2016. Microbial enzymes: industrial progress in $21^{\text {st }}$ century. 3 Biotech 6: 174.

31. Iyer PV, Ananthanarayan L. 2008. Enzyme stability and stabilization - aqueous and non-aqueous environment. Process Biochem. 43: 1019-1032.

32. Sharma S, Pathak N, Chattopadhyay K. 2012. Osmolyte induced stabilization of protein molecules: a brief review. J. Proteins Proteomics 3: 129-139.

33. Lin LL, Chou PR, Hua YW, Hsu WH. 2006. Overexpression, one-step purification, and biochemical characterization of a recombinant $\gamma$-glutamyl transpeptidase from Bacillus licheniformis. Appl. Microbiol. Biotechnol. 73: 103-112.

34. Kelly SM, Jess TJ, Price NC. 2005. How to study proteins by circular dichroism. Biochim. Biophys. Acta 1751: 119-139.

35. Greenfield NJ. 2004. Analysis of circular dichroism data. Methods Enzymol. 383: 282-317.

36. Royer CA. 2006. Probing protein folding and conformational transitions with fluorescence. Chem. Rev. 106: 1769-1784.

37. Yang JC, Liang WC, Chen YY, Chi MC, Lo HF, Chen HL, et al. 2011. Biophysical characterization of Bacillus licheniformis and Escherichia coli $\gamma$-glutamyl transpeptidases: a comparative analysis. Int. J. Biol. Macromol. 48: 414-422.

38. Chi MC, Lo YH, Chen YY, Lin LL, Merlino A. 2014. $\gamma$-Glutamyl transpeptidase architecture: effect of extra sequence deletion on autoprocessing, structure and stability of the protein from Bacillus licheniformis. Biochim. Biophys. Acta 1844: 2290-2297.

39. Lin LL, Chen YY, Chi MC, Merlino A. 2014. Low-resolution $X$-ray structure of $\gamma$-glutamyl transpeptidase from Bacillus licheniformis: opened active site cleft and a cluster of acid residues potentially involved in the recognition of a metal ion. Biochim. Biophys. Acta 1844: 1523-1529.

40. Lin MG, Chi MC, Chen YY, Wang TF, Lo HF, Lin LL 2016.
Site-directed mutagenesis of a conserved Asn450 residue of Bacillus licheniformis $\gamma$-glutamyl transpeptidase. Int. J. Biol. Macromol. 91: 416-425.

41. Klibanov AM. 1983. Stabilization of enzymes against thermal inactivation. Adv. Appl. Microbiol. 29: 1-28.

42. Hu HY, Yang JC, Chen JH, Chi MC, Lin LL. 2012. Enzymatic characterization of Bacillus licheniformis $\gamma$-glutamyl transpeptidase fused with N-terminally truncated forms of Bacillus sp. TS23 a-amylase. Enzyme Microb. Technol. 51: 86-94.

43. Ptitsyn OB. 1995. Molten globule and protein folding. Adv. Protein Chem. 47: 83-229.

44. Chi MC, Chen YY, Lo HF, Lin LL. 2012. Experimental evidence for the involvement of amino acid residue Glu398 in the autocatalytic processing of Bacillus licheniformis $\gamma$ glutamyl transpeptidase. FEBS Open Bio 2: 298-304.

45. Lakowicz JR. 2006. Protein fluorescence. In: Principles of Fluorescence Spectroscopy, pp 529-575, Springer, Boston, MA.

46. Yancey PH, Somero GN. 1980. Methylamine osmoregulatory solutes of elasmobranch fishes counteract urea inhibition of enzymes. J. Exp. Zool. 212: 1214-1222.

47. Timasheff SN. 1993. The control of protein stability and association by weak interactions with water: how do solvents affect these processes? Ann. Rew. Biophys. Biomol. Struct. 22: 67-97.

48. Zou Q, Bennion BJ, Daggett V, Murphy KP. 2002. The molecular mechanism of stabilization of proteins by TMAO and its ability to counteract the effect of urea. J. Am. Chem. Soc. 54: 4125-4139.

49. Arakawa T, Timasheff SN. 1982. Stabilization of protein structure by sugars. Biochemistry 21: 6536-6544.

50. Garrett JM, Stairs RA, Annett RG. 1988. Thermal denaturation and coagulation of whey proteins: effect of sugars. J. Dairy Sci. 71: 10-16.

51. Oshima H, Kinoshita M. 2013. Effects of sugars on the thermal stability of a protein. J. Phys. Chem. 138: 245101.

52. Graziano G. 2012. How does sucrose stabilize the native state of globular proteins? Int. J. Biol. Macromol. 50: 230-235.

53. Street TO, Bolen DW, Rose DW. 2006. A molecular mechanism for osmolyte-induced protein stability. Proc. Natl. Acad. Sci. USA 103: 13997-14002.

54. Holthauzen LM, Rösgen J, Bolen DW. 2010. Hydrogen bonding progressively strengthens upon transfer from the protein urea-denatured state to water and protecting osmolytes. Biochemistry 49: 1310-1318.

55. Timasheff SN. 2002. Protein-solvent preferential interactions, protein hydration and the modulation of biochemical reactions by solvent components. Proc. Natl. Acad. Sci. USA 99: 97219726.

56. Singh R, Haque I, Ahmad F. 2005. Counteracting osmolyte trimethylamine $\mathrm{N}$-oxide destabilizes proteins at $\mathrm{pH}$ below its $p$ Ka. J. Biol. Chem. 280: 1035-1042.

57. Santoro MM, Liu Y, Khan SMA, Hou LX, Bolen DW. 1992. Increased thermal stability of proteins in the presence of 
naturally occurring osmolytes. Biochemistry 31: 5278-5283.

58. Kaushik JK, Bhat R. 1998. Thermal stability of proteins in aqueous polyol solutions: role of the surface tension of water in the stabilizing effect of polyols. J. Phys. Chem. 102: 7058-7066.

59. Bruździak P, Panuszko A, Jourdan M, Stangret J. 2016. Protein thermal stabilization in aqueous solutions of osmolytes. ActaBiochim. Pol. 63: 65-70.

60. Liu Y, Bolen DW. 1995. The peptide backbone plays a dominant role in protein stabilization by naturally occurring osmolytes. Biochemistry 34: 12884-12891.

61. Tsou CL. 1995. Inactivation precedes overall molecular conformation changes during enzyme denaturation. Biochim. Biophys. Acta 1253: 151-162.

62. Bennion BJ, Daggett V. 2003. The molecular basis for the chemical denaturation of proteins by urea. Proc. Natl. Acad. Sci. USA 100: 5142-5147.

63. Taylor LS, York P, Williams AC, Edwards HC, Mehta V, Jackson GS, et al. 1995. Sucrose reduces the efficiency of protein denaturation by a chaotropic agent. Biochim. Biophys. Acta 1253: 39-46.

64. Chen BL, Arakawa T. 1996. Stabilization of recombinant human keratinocyte growth factor by osmolytes and salts. J. Pharm. Sci. 85: 419-426.

65. Ou WB, Park YD, Zhou HM. 2001. Molecular mechanism for osmolyte protection of creatine kinase against guanidine denaturation. Eur. J. Biochem. 268: 5901-5911.

66. Kim YS, Jones LS, Dong A, Kendrick BS, Chang BS, Manning MC, et al. 2003. Effects of sucrose on conformational equilibria and fluctuations within the native-state ensemble of proteins. Protein Sci. 12: 1252-1261.

67. Wang A, Bolen DW. 1997. A naturally occurring protective system in urea-rich cells: mechanism of osmolyte protection of protein against urea denaturation. Biochemistry 36: 9101-9108.

68. Burg MB, Ferraris JD. 2008. Intracellular organic osmolytes: function and regulation. J. Biol. Chem. 283: 7309-7313.

69. Almarza J, Rincon L, Bahsas A, Brito F. 2009. Molecular mechanism for the denaturation of proteins by urea. Biochemistry 48: 7608-7613.

70. Hua L, Zhou R, Thirumalai D, Berne BJ. 2008. Urea denaturation by stronger dispersion interactions with proteins than water implies a 2-stage unfolding. Proc. Natl. Acad. Sci. USA 105: 16928-16933.

71. Singh LR, Poddar NK, Dar TA, Kumar R, Ahmad F. 2011. Protein and DNA destabilization by osmolytes: the other side of the coin. Life Sci. 88: 117-125.

72. Kelly RH, Yancey PH. 1999. High contents of trimethylamine oxide correlating with depth in deep-sea teleost fishes, skates, and decapod crustaceans. Biol. Bull. 196: 18-25.

73. Venkatesu P, Lee MJ, Lin HM. 2009. Osmolyte counteracts urea-induced denaturation of $\alpha$-chymotrypsin. J. Phys. Chem. 113: $5327-5338$. 